\title{
Optimization of the distance between the rows in solar collectors' arrays
}

\author{
Merima Zlateva ${ }^{1^{*}}$ \\ ${ }^{1}$ Technical University of Sofia, Department of Heating and Refrigeration Engineering, 1000 Sofia, \\ Bulgaria
}

\begin{abstract}
The article presents some results of the analysis of the influence of the distance between the collectors' rows in thermal solar systems on the degree of self-shading. For the purposes of the study, the dimensionless ratio Ks between the required minimum distance between the rows, guaranteeing the absence of self-shading, and the height of the collectors, was used. A simulation study was performed for specific climatic conditions and the annual solar radiation incident on the collectors' array at different degrees of self-shading was determined. Its annual financial equivalent for various alternative heat sources (electricity, natural gas, wood pellets, district heating and gas oil) was assessed, as well as the necessary investment costs for construction of the solar system. A regression relationship between the coefficient of net present value NPVQ and Ks was derived and its optimal values were established, which ensure the achievement of maximum economic efficiency of the system operation for the considered replaced heat sources.
\end{abstract}

\section{Introduction}

Solar energy is utilized via thermal [1] and photovoltaic solar systems. In solar systems designed to provide large capacities it is necessary to divide the collector's array in more than one row. Often in such cases, the available surface on the building roof or the ground is limited and shading between the rows is inevitable. In such circumstances, determining the degree of self-shading of all rows behind the first one is a prerequisite for correct assessment of the solar radiation incident on the array. This applies to both thermal and photovoltaic solar systems [2].

In [3] is presented a mathematical optimization procedure for thermal solar systems, used to obtain the field and collectors' design parameters (number of rows, distance between collector rows, collector height, and collector inclination angle). The aim of the optimisation procedure is to minimize the periodic cost of a solar plant, producing a given amount of annual energy, and the unit cost of energy. The optimal deployment of the collectors is presented as a function of daily energy demand, with the cost of land and collector efficiency as parameters.

\footnotetext{
* Corresponding author: mzlat@,tu-sofia.bg
} 
The degree of self-shading depends on the distance between the rows $R$, the height of the collectors $L$, their slope angle to the horizon $\beta$, the latitude $\varphi$ and the position of the sun, defined by the solar altitude $\alpha_{s}$ and azimuth $\gamma_{s}$ angles (Fig. 1). According to data from various sources, e.g. [3, 4], the length of the rows does not have a noticeable effect on the solar radiation falling on the collectors' array, which is confirmed by the author's research, carried out with the simulation software Transol [5].

\section{Theoretical considerations}

The parameters characterizing the mutual position of two adjacent collectors' rows and necessary for estimating the degree of self-shading for southern orientation are shown in Fig. 1. To ensure maximum irradiation of the surface at a certain solar attitude, the angle $v$ between the horizon and the line connecting the upper and lower edges of two successive collectors' rows have to meet the following condition:

$$
v \leq \alpha_{s}
$$

The solar altitude $\alpha_{s}$ is determined by the ratio of the lengths of the sides of the triangles in Fig. 1 end expressed by (2):

$$
\operatorname{tg} \alpha_{s}=h \cdot b^{-1}
$$

Where $b$ and $h$ are the projections on the horizontal and vertical surfaces of the line connecting the upper and lower edges of the successive collectors' rows.

For distance between the rows $R=a+b$ and collector height $L$, the following trigonometric dependences are valid:

$$
\begin{gathered}
\sin \beta=\frac{h}{L} \Rightarrow h=L \cdot \sin \beta \\
\cos \beta=\frac{a}{L}=\frac{R-b}{L} \Rightarrow R-b=L \cdot \cos \beta \Rightarrow b=R-L \cdot \cos \beta
\end{gathered}
$$

Fig 1. Shading of south-facing collectors' rows.

After substituting (3), (4) and (2) in (1), the condition of absence of self-shading of two consecutive rows can be presented with (5) and is reduced to determining the minimum 
allowable value of $R$, expressed as a linear function of the height of the collectors $L$, the angle $\beta$ and the tangent of the angle $v=f\left(\alpha_{s}\right)$ :

$$
R \geq L \cdot\left(\cos \beta+\frac{\sin \beta}{\operatorname{tg} v}\right)
$$

In [6] the dimensionless criterion $K_{S}(\tau)$ is defined as a function of time $\tau$ :

$$
K_{S}(\tau)=R_{l}^{\min }(\tau) \cdot L^{-1}
$$

The criterion $K_{S}(\tau)$ is interpreted as a coefficient of proportionality between the required minimum distance between the rows of the collectors' array $R_{1}^{\min }(\tau)$, ensuring the absence of self-shading and the height of the collectors $L$. For $K_{S}(\tau)$ the author has derived a generalized functional dependence on the type:

$$
K_{s}(\varphi, \beta)=\left.a \cdot \varphi^{b} \cdot \beta^{c}\right|_{m=c o n s t}
$$

Where $m$ is the month of the year; $a, b$ and $c$ are constants the monthly values of which depend on the latitude $\varphi$ and the angle $\beta$.

The geographical location of Bulgaria, with a relatively small range of variation of $\varphi$, does allow simplification of dependence (7) and its representation as a function only of the angle $\beta$. In Fig. 2 is shown the change of the parameter $K_{s}$ for the months from November to February, for values of the angle of inclination of the collectors in the range $\beta=20^{\circ} \div 45^{\circ}$. The graph is constructed using (7) for the medium latitude of Bulgaria with relative error $<1,5 \%$

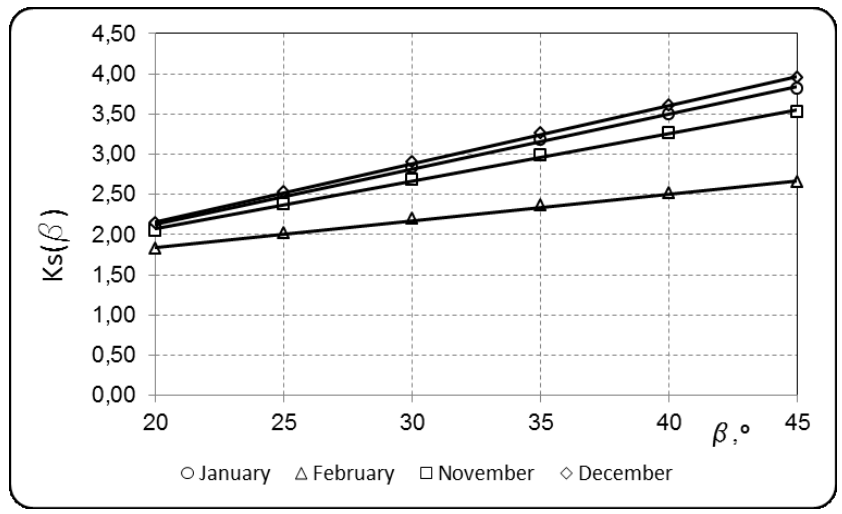

Fig. 2. Coefficient of proportionality $K_{s}(\beta)$.

The fulfillment of (8) for the month of December, during which the solar altitude angle has minimum values, is a condition for year-round prevention of self-shading of the collectors' rows:

$$
R_{1}^{\text {min }} \geq L \cdot K_{S}(\beta)
$$


The decrease in the ratio $K_{s}$ causes an increase in the shading which has a significant effect mainly on the direct solar radiation, as its maximum instantaneous values are in the hours immediately after sunrise and before sunset during the winter, when the intensity of the solar radiation is low. However, with a certain area available for the collectors' array, the reduction of $K_{S}$ allows the placement of a larger number of collectors' rows and an increase of the annual solar radiation, despite the negative effect of self-shading. This gives reason for different authors, e. g. [3], to look for the optimal distance between the collectors' rows based on energy and economic criteria.

\section{Optimal distance between the rows in solar collectors' arrays}

The annual solar radiation falling on a collectors' array at different degrees of self-shading is determined with the help of the software Transol [5] for the climatic conditions of the city of Varna. An array with 8 flat-plate collectors is considered as a reference. The collectors' parameters are as follows: height $L=2,07 \mathrm{~m}$, width $B=1,0 \mathrm{~m}$, optical efficiency $\eta_{0}=0,82$ and heat loss coefficient $a_{0}=3,65$, slope angle $\beta=43^{\circ}$. To avoid shading, it is necessary to fulfil the condition $K_{S}=4$ and the required area of the terrain is $A_{c a, K_{s}^{*}=4}=39,2, \mathrm{~m}^{2}$.

As an alternative, collectors' arrays with a different degree of self-shading are considered, for which the condition is fulfilled $A_{c a, K_{s}=v a r} \leq A_{c a, K_{S}=4}\left(A_{c a, K_{S}=v a r}\right.$ surface of the terrain occupied by the alternative collectors' arrays).

The annual solar radiation on the collectors' array $H_{s h, a}, M W h / a$ with $K_{S}=[1 ; 1.5 ; 2 ; 2.5 ; 4]$ is shown on Fig.3. The annual financial equivalent of the incident solar radiation, determined for different alternative heat sources $B=f\left(K_{S}\right)$, taking into account the efficiency of the system, is shown in Fig. 4.

The results presented in Fig. 4 are obtained according to the typical prices of the replaced energy sources [8]. The equivalent annualized value $I_{a}\left(K_{S}\right)$ of the investment costs for the solar system is also shown.

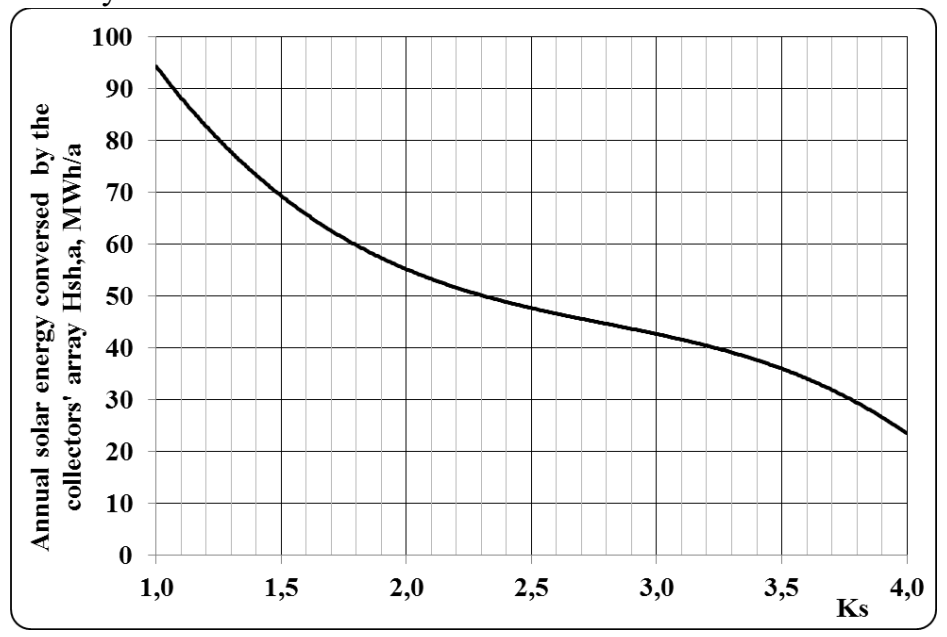

Fig. 3. Annual solar energy conversed by the collector array. 
The presented dependencies $B=f\left(K_{s}\right)$ and $I_{a}\left(K_{s}\right)$ clearly illustrate the different rates of change of each. This allows to formulate the hypothesis that there is an optimal value of the criterion "net present value ratio", defined by (9) and showing the net income from the operation of the system for an investment of BGN 1.

$$
N P V Q=\frac{N P V}{I\left(K_{s}^{*}\right)}
$$

Where $N P V=B \frac{1-(1+r)^{-n}}{r}-I_{a}$ is the net present value, BGN;

$B$ - financial equivalent of the energy recovered by the solar system, BGN;

$I_{a}$ - annuitizes investment costs, BGN;

$n$ - time period of the analysis;

$r$ - discount rate.

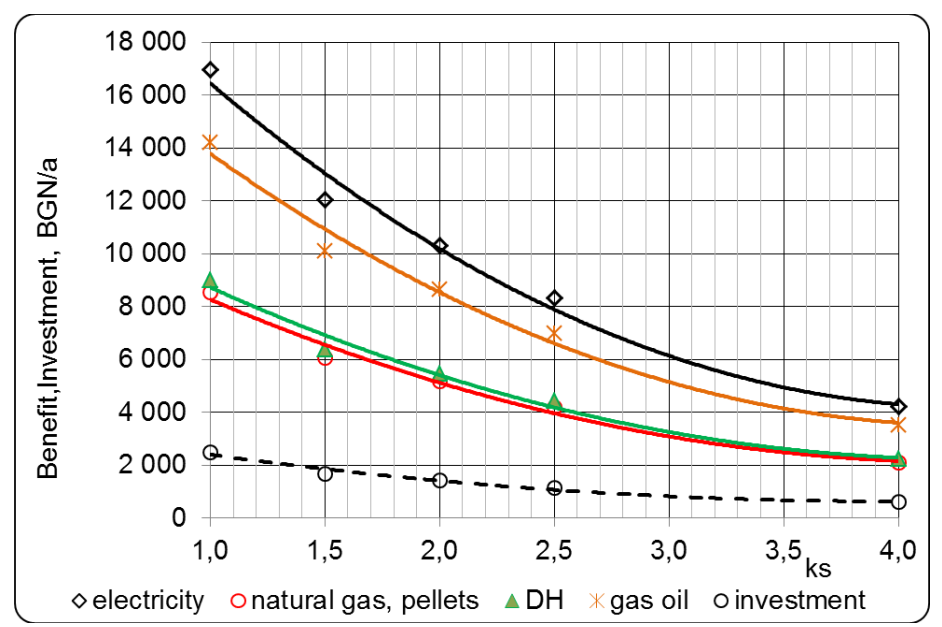

Fig. 4. Benefit and annual investment cost for the solar system.

The net present value ratio for the different types of substitute heat sources at values of $K_{s}$ in the range $K_{S}^{*}=[1 \div 4]$ is shown in Fig. 5. The obtained regression dependencies $N P V Q=f\left(K_{s}^{*}\right)$ were studied in order to find the optimal values of the ratio $K_{s, o p t}$ at which the maximum value of $N P V Q=f\left(K_{S}\right)$ is achieved. 


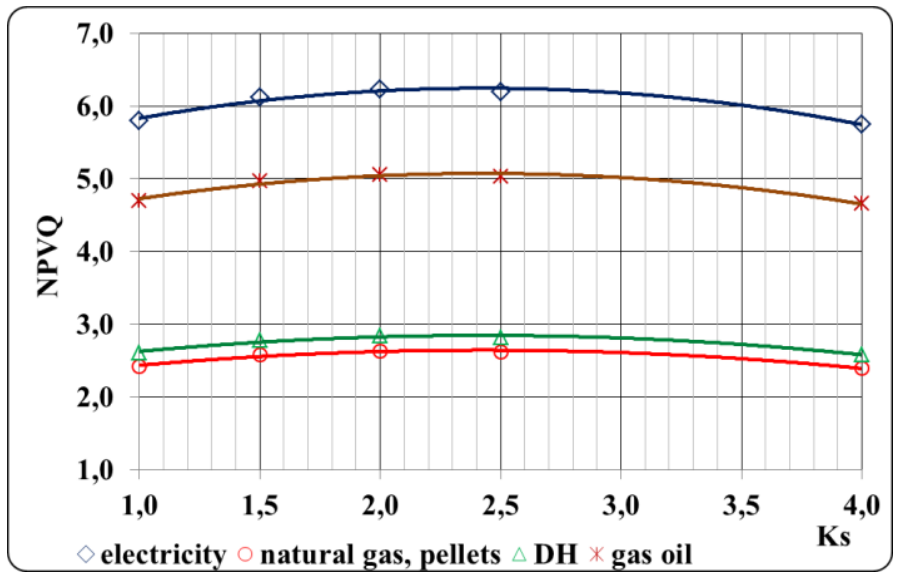

Fig. 5. Net present value ratio depending on the factor $K_{S}$.

The functional dependences $N P V Q=f\left(K_{S}\right)$ are summarized in Table 1. The table also shows the values of the ratio $K_{s, o p t}$.

Table 1. Functional dependence and optimal values of $N P V Q=f\left(K_{S}\right)$.

\begin{tabular}{|l|c|c|c|}
\hline & Functional dependence & $N P V Q \max$ & $K_{s, \text { opt }}$ \\
\hline Electricity & $N P V Q=-0,16 \cdot K_{s}^{2}+0,79 \cdot K_{s}+3,84$ & 6,03 & 2,5 \\
\hline Gas oil & $N P V Q=-0,14 \cdot K_{s}^{2}+0,66 \cdot K_{s}+3,06$ & 4,85 & 2,4 \\
\hline District heating & $N P V Q=-0,09 \cdot K_{s}^{2}+0,42 \cdot K_{s}+1,57$ & 2,70 & 2,3 \\
\hline Natural gas, pellets & $N P V Q=-0,08 \cdot K_{s}^{2}+0,40 \cdot K_{s}+1,43$ & 2,54 & 2,5 \\
\hline
\end{tabular}

\section{Conclusions}

The study shows that with the accepted financial parameters and prices of the replaced energy sources, the functional dependencies $N P V Q=f\left(K_{S}^{*}\right)$ are approximated with a high degree of accuracy (multiple correlation coefficients $R^{2}=0,97$ ) by second degree polynomials.

In the studied range of change of the ratio $K_{S}=[1 \div 4]$, the net present value ratio $N P V Q>1$, which guarantees the efficiency of the financial resources invested for the construction of the system. For the considered energy sources, its maximum values are achieved at $K_{s, o p t}=2,5$ for electricity, natural gas and pellets; $K_{s, o p t}=2,4$ for gas oil and $K_{s, o p t}=2,3$ in case of energy from district heating.

Despite the small range of values of $K_{s, o p t}$, the maximum values of the net present value ratio $N P V Q_{\max }$ vary considerably: 6.03 for electricity; 4.85 for gas oil; 2.7 for district heating and 2.54 for natural gas and pellets. 
For the efficient operation of thermal solar systems, it is necessary, right at the design stage, to determine correctly the degree of shading of the collectors' array. The optimal distance between the rows must be determined on the basis of energy and economic criteria, taking into account the specific financial conditions.

\section{References}

1. N. Kaloyanov, L. Tsokov, R. Tsekov, M. Vassilev, Ts. Bozhkov. Proceedings of the XXIII Scientific Conference with International Participation FPEPM 2018 (in Bulgarian), pp. $72-77$, (2018)

2. S. Omidreza et al., Malaysia Advances in Environment, Biotechnology and Biomedicine, pp 100 - 106 (2012)

3. D. Weinstock, J. Appelbaum, Jour. of Sol. En. Eng., 129, pp 363 - 370 (2007)

4. S. Stamov et al., Handbook on Heating, Air conditioning and Cooling. Part II. Heating, heat and gas supply, (2001)

5. Sistems Avancats d'Energia Solar Termica Transol for Windows

6. J. A. Duffie, W Beckman, Solar Engineering of Thermal Processes, (2013)

7. N. Kaloyanov, Analysis and synthesis of low-potential combined systems, (1989) 